\title{
Bigger perturbations enhance higher trophic levels biomass, increase transfer efficiency and may sustain for bigger plankton biodiversity
}

\author{
Anupam Priyadarshi ${ }^{1, *}$ and Ram Chandra ${ }^{1, * *}$ \\ ${ }^{1}$ Department of Mathematics, Banaras Hindu University, Varanasi-221005 India
}

\begin{abstract}
Highly intermittent phytoplankton is ubiquitously observed when measurements are performed at micro-scale $(<1 \mathrm{~mm})$. The conventional way of plankton modelling is based on the mean-field approach in which only the first central-moment approximations is retained and ignored higher central moments). The conventional modeling approach may be suitable for meso- or bigger scale $(\mathrm{km})$ but it is inappropriate for micro-scale $(<1 \mathrm{~mm})$ where observed overlap in the intermittent spatial distributions of predators and prey become more important for determining the flow of nutrients and energy up the food chain. A new modelling approach called closure modelling is developed to account intermittent phytoplankton using Reynold's decomposition from turbulence theory and retaining higher central moment approximations in Taylor series. In this study, we developed a NPZD compartmental model to describe the interactions of nutrient $(\mathrm{N})$, phytoplankton $(\mathrm{P})$, zooplankton $(\mathrm{Z})$ and detritus (D) using closure modelling which accounts mean and fluctuating parts of these plankton variables. The results obtained in NPZD compartmental model confirm that perturbation / heterogeneity supports higher trophic levels involved in the model. This reassured the earlier results observed in case of NP and NPZ models in which perturbations enhances P-biomass and Z-biomass respectively. It is observed that perturbation / heterogeneity and a statistical quantity called coefficient of variations of phytoplankton $\left(C V_{p}\right)$ (ratio of standard deviation and mean) are positively associated in plankton ecosystems. The perturbations / heterogeneity leads to higher transfer efficiency (Z-biomass/P-biomass) in plankton ecosystems. These results are robust i.e. independent of parameters choices. Perturbation / heterogeneity effects on community structure, species richness and may quantify the energy transfer along trophic levels through biological process from primary production to higher trophic levels. Based on our study, we hypothesize that the locations with high $\left(C V_{p}\right)$ are highly heterogeneous and have high transfer efficiency, while low $\left(C V_{p}\right)$ locations are less heterogeneous around Tokyo Bay.
\end{abstract}

\section{Introduction}

Plankton are the productive base of the aquatic ecosystem and assumed to control climate change globally as they build up organic compounds directly from carbon dioxide $\mathrm{CO}_{2}$ and

\footnotetext{
*e-mail: anupam240@gmail.com

**e-mail: rchandrabhu25@gmail.com
} 
dissolved nutrient in waters. The competition, excretion, respiration and mineralization are the potentially effective processes which may induced global changes in plankton communities and their complex interactions between biotic and abiotic components. Such interactions ensure the progress in plankton communities through processes photosynthesis, predation, excretion, respiration and remineralization under which dynamics of marine systems can be estimated. The evolution of these interactions may bring changes in management of marine systems and global impact of ecological consequences. Several experimental studies confirmed the variation in phytoplankton biomass with abiotic components such as nutrient and light, across the distribution scale micro- to meso- to kilometre ([1], [2], [3], [4], [5], [6],[7], [8]). Based on experimental and observational studies on plankton systems, theoretical framework of plankton modelling are developed to validate observed data and to predict ecological processes. From nutrient-phytoplankton (NP) interaction models to NPZDF compartmental models are developed based on mean-field approach, which may be appropriate for mesoor bigger scale plankton distribution but not suitable where fluctuations against mean dominate. Recent measurements perform at micro-scale confirmed the ubiquitous presence of intermittent phytoplankton distributions at micro-scale $(<1 \mathrm{~mm})([4],[9],[10],[11],[12]$, [13]). Detritus in plankton ecosystems play a vital role in energy dissipation and remineralization process through microbial activities. Recycling of minerals through microbial activity enhances primary productions and supports as trophic resource for higher trophic levels. The loss of phytoplankton and zooplankton sinks the biomass which become resource of nutrients and detritus. The detritus breaks dead organic materials into utilizable nutrients in plankton system. Being explicitly added detritus into NPZ system, increases compartments and hence, complexity of the model which may potentially affect the earlier studies results on NP and NPZ models. We are interested to observe the NPZD plankton dynamics when complexity increase in models by adding detritus explicitly into NPZ models.

Closure modelling is developed using Reynolds decomposition and truncated Taylors series (retaining up to second order terms) to account fluctuation in observed plankton data. A statistical quantity called coefficient of variation of phytoplankton (CVp) (standard against plankton mean) at several locations of Tokyo Bay is calculated from observed data which is measured using TurboMap Laser ([4], [6], [12]) at micro-scale (mm-scale). The measured quantity $\mathrm{CVp}$ is used to calculate (estimate) perturbation / heterogeneity $\beta$ at those locations which is ratio of total fluctuation strength to total mean square i.e.,

$$
\begin{array}{r}
\text { perturbation/heterogeneity }(\beta)=\frac{\text { Total Flctuations }}{(\text { mean })^{2}} \\
\beta=\frac{\Sigma \text { variances }+2 \Sigma \text { covarinces }}{(\Sigma \text { Mean })^{2}}
\end{array}
$$

Perturbations / Heterogeneity is known to increase richness ([10], [14]) and sustain higher diversity ([15], [16],[17]). Our observations in this study hypothesized that in the plankton ecosystems, perturbation / heterogeneity positively associated with higher overdiversity and higher transfer efficiency. Transfer efficiency (TE) plays important roles in management of organic substance and to change the rate of functioning processes between producers and consumers which affect community structure and biomass productivity within the ecosystems. Transfer efficiency is a way to measure the energy transfer within trophic level and defined as the ratio of production of zooplankton biomass to the phytoplankton production biomass ( [15], [16], [18]). TE may influence the trophic interactions from resource to consumer and generally, high TE regarded to have higher influence on trophic interactions. The extensive simulations are carried out to address the following pertinent questions:

1. How perturbations affect trophic levels, in particular higher trophic level? 
2. How perturbations associated with observed $C V_{p}$ across the gradient of Tokyo Bay?

3. How perturbation affect Transfer efficiency in plankton ecosystems?

\section{Model Formulation}

\subsection{Conventional NPZD model}

Assuming $\mathrm{N}$ as a nutrient, $\mathrm{P}$ as phytoplankton biomass, $\mathrm{Z}$ as zooplankton biomass and $\mathrm{D}$ as detritus biomasses are available in plankton ecosystem. A conventional NPZD model based on mean-field approach is developed below:

$$
\begin{aligned}
\frac{d N}{d t} & =-v_{\max } \frac{N}{K+N} P+M_{Z N} Z+\gamma_{M} D \\
\frac{d P}{d t} & =v_{\max } \frac{N}{K+N} P-M_{P} P-\frac{R P^{2}}{K_{P}^{2}+P^{2}} Z \\
\frac{d Z}{d t} & =(1-\gamma) \frac{R P^{2}}{K_{P}^{2}+P^{2}} Z-M_{Z N} Z-M_{Z D} D \\
\frac{d D}{d t} & =\gamma \frac{R P^{2}}{K_{P}^{2}+P^{2}} Z+M_{P} P+M_{Z D} D-\gamma_{M} D
\end{aligned}
$$

Here, phytoplankton biomass depends on

(i) nutrient uptake by phytoplankton $v_{\max } \frac{N}{K+N} P$, where $v_{\max }$ is phytoplankton response to light and $\frac{N}{K+N}$ is Monod function ( $K$ is half-saturation constant) used for nutrient uptake by phytoplankton.

(ii) consumption of microalgae by zooplankton according to Holling type III $\frac{R P^{2}}{K_{P}^{2}+P^{2}}$ grazing functional response where is specific grazing rate and $K_{P}$ is half-saturation constant.

(iii) phytoplankton natural mortality except for small assimilation which assimilated into detritus. Zooplankton grow by grazing phytoplankton while linear mortality is transfer to nutrient and detritus respectively.

Detritus remineralized by microbes with fraction $\gamma_{M} D$ into nutrient compartment. The NPZD model have been developed conventionally (Fig. 1) by retaining only first central moments ignoring higher central moments ([19], [20], [21], [22]).

\subsection{Closure Model}

Using the Reynolds decomposition and Taylor series around the mean values as

$$
\begin{array}{rrr}
N(s, t)=N_{0}(s, t)+N^{\prime}(s, t) & P(s, t)= & P_{0}(s, t)+P^{\prime}(s, t) \\
Z(s, t)=Z_{0}(s, t)+Z^{\prime}(s, t) & D(s, t)= & D_{0}(s, t)+D^{\prime}(s, t)
\end{array}
$$

Where $N_{0}, P_{0}, Z_{0}$ and $D_{0}$ are the spatial means of biomasses and $N^{\prime}, P^{\prime}, Z^{\prime}$ and $D^{\prime}$ are their respective fluctuating components. Assuming at a particular time, the spatial average of the fluctuating parts is zero i.e. mean value equals to spatial average of each variables and taking Taylors series expansion up to second order only, the temporal equations of mean, variance 


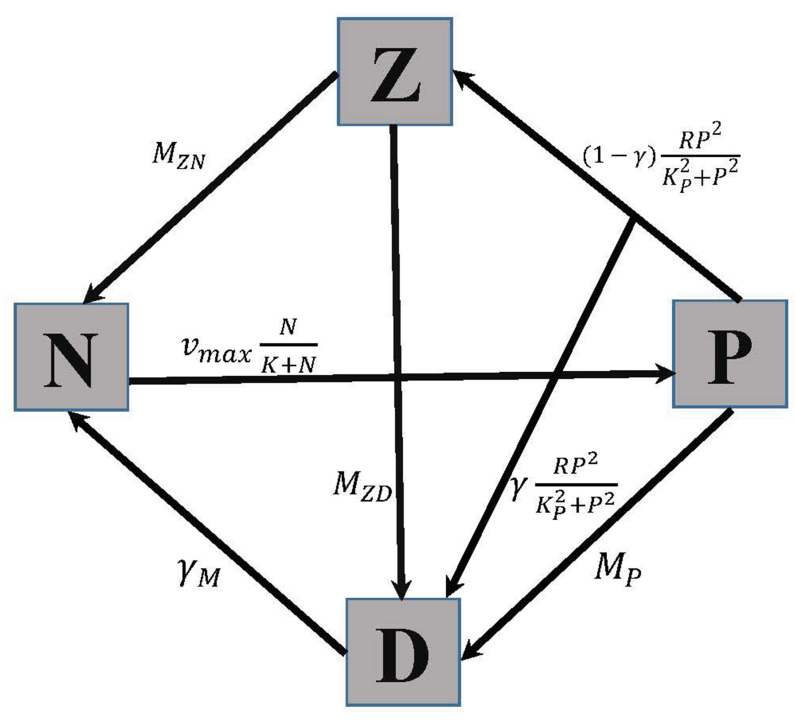

Figure 1. Schematic diagram of energy flow-up in the NPZD system

and covariance are derived. Using the following scaled variables and parameters, the developed NPZD closure model transformed into the dimensionless form (reduced parameters and equations):

$$
\begin{gathered}
n_{0}=\frac{N_{0}}{A}, \quad p_{0}=\frac{P_{0}}{A}, \quad z_{0}=\frac{Z_{0}}{A}, \quad d_{0}=\frac{D_{0}}{A} \\
\left\langle n^{\prime 2}\right\rangle=\frac{\left\langle N^{\prime 2}\right\rangle}{B},\left\langle p^{\prime 2}\right\rangle=\frac{\left\langle P^{\prime 2}\right\rangle}{B}, \quad\left\langle z^{\prime 2}\right\rangle=\frac{\left\langle Z^{\prime 2}\right\rangle}{B}, \quad\left\langle d^{\prime 2}\right\rangle=\frac{\left\langle D^{\prime 2}\right\rangle}{B} \\
\left\langle n^{\prime} p^{\prime}\right\rangle=\frac{\left\langle N^{\prime} P^{\prime}\right\rangle}{B},\left\langle n^{\prime} z^{\prime}\right\rangle=\frac{\left\langle N^{\prime} Z^{\prime}\right\rangle}{B},\left\langle p^{\prime} z^{\prime}\right\rangle=\frac{\left\langle P^{\prime} Z^{\prime}\right\rangle}{B},\left\langle p^{\prime} d^{\prime}\right\rangle=\frac{\left\langle P^{\prime} D^{\prime}\right\rangle}{B},\left\langle z^{\prime} d^{\prime}\right\rangle=\frac{\left\langle Z^{\prime} D^{\prime}\right\rangle}{B} \\
r=\frac{R}{v_{\max }}, m_{p}=\frac{M_{P}}{v_{\max }}, m_{z n}=\frac{M_{Z N}}{v_{\max }}, m_{z d}=\frac{M_{Z D}}{v_{\max }}, \gamma_{m}=\frac{\gamma_{M}}{v_{\max }}, k=\frac{K}{A}, k_{p}=\frac{K_{P}}{A} \\
\beta=\frac{\left\langle N^{\prime 2}\right\rangle+\left\langle P^{\prime 2}\right\rangle+\left\langle Z^{\prime 2}\right\rangle+\left\langle D^{\prime 2}\right\rangle+2\left(\left\langle N^{\prime} P^{\prime}\right\rangle+\left\langle N^{\prime} Z^{\prime}\right\rangle+\left\langle N^{\prime} D^{\prime}\right\rangle+\left\langle P^{\prime} Z^{\prime}\right\rangle+\left\langle P^{\prime} D^{\prime}\right\rangle+\left\langle Z^{\prime} D^{\prime}\right\rangle\right)}{\left(N_{0}+P_{0}+Z_{0}+D_{0}\right)^{2}}
\end{gathered}
$$

After introducing the scaled variables, scalar quantities and perturbation $\beta$ (from 7) in the closure NPZD model, the following relations hold:

$$
\begin{gathered}
n_{0}+p_{0}+z_{0}+d_{0}=1 \\
\left\langle n^{\prime 2}\right\rangle+\left\langle p^{\prime 2}\right\rangle+\left\langle z^{\prime 2}\right\rangle+\left\langle d^{\prime 2}\right\rangle+2\left(\left\langle n^{\prime} p^{\prime}\right\rangle+\left\langle n^{\prime} z^{\prime}\right\rangle+\left\langle n^{\prime} d^{\prime}\right\rangle+\left\langle p^{\prime} z^{\prime}\right\rangle+\left\langle p^{\prime} d^{\prime}\right\rangle+\left\langle z^{\prime} d^{\prime}\right\rangle\right)=1
\end{gathered}
$$

The temporal variations of mean in NPZD model are described below: 


$$
\begin{aligned}
\frac{d p_{0}}{d t}= & \frac{n_{0}}{k+n_{0}} p_{0}-\frac{k p_{0}}{\left(k+n_{0}\right)^{3}} \beta\left\langle n^{\prime 2}\right\rangle+\frac{k}{\left(k+n_{0}\right)^{2}} \beta\left\langle n^{\prime} p^{\prime}\right\rangle-m_{p} p_{0} \\
& -r\left[\frac{p_{0}^{2} z_{0}}{k_{p}^{2}+p_{0}^{2}}+\frac{k_{p}^{2} z_{0}\left(k_{p}^{2}-3 p_{0}^{2}\right)}{\left(k_{p}^{2}+p_{0}^{2}\right)^{3}} \beta\left\langle p^{\prime 2}\right\rangle+\frac{2 k_{p}^{2} p_{0}}{\left(k_{p}^{2}+p_{0}^{2}\right)^{2}} \beta\left\langle p^{\prime} z^{\prime}\right\rangle\right] \\
\frac{d z_{0}}{d t}= & (1-\gamma) r\left[\frac{p_{0}^{2} z_{0}}{k_{p}^{2}+p_{0}^{2}}+\frac{k_{p}^{2} z_{0}\left(k_{p}^{2}-3 p_{0}^{2}\right)}{\left(k_{p}^{2}+p_{0}^{2}\right)^{3}} \beta\left\langle p^{\prime 2}\right\rangle+\frac{2 k_{p}^{2} p_{0}}{\left(k_{p}^{2}+p_{0}^{2}\right)^{2}} \beta\left\langle p^{\prime} z^{\prime}\right\rangle\right] \\
& -\left(m_{z n}+m_{z d}\right) z_{0} \\
\frac{d d_{0}}{d t}= & \gamma r\left[\frac{p_{0}^{2} z_{0}}{k_{p}^{2}+p_{0}^{2}}+\frac{k_{p}^{2} z_{0}\left(k_{p}^{2}-3 p_{0}^{2}\right)}{\left(k_{p}^{2}+p_{0}^{2}\right)^{3}} \beta\left\langle p^{\prime 2}\right\rangle+\frac{2 k_{p}^{2} p_{0}}{\left(k_{p}^{2}+p_{0}^{2}\right)^{2}} \beta\left\langle p^{\prime} z^{\prime}\right\rangle\right] \\
& +m_{z d} z_{0}-\gamma_{m} d_{0}+m_{p} p_{0}
\end{aligned}
$$

The variance equations of NPZD closure model are given below

$$
\begin{aligned}
\frac{\left\langle n^{\prime 2}\right\rangle}{d t}= & -2\left[\frac{n_{0}}{k+n_{0}}\left\langle n^{\prime} p^{\prime}\right\rangle-\frac{k p_{0}}{\left(k+n_{0}\right)^{2}}\left\langle n^{\prime 2}\right\rangle\right]+2 m_{z n}\left\langle n^{\prime} z^{\prime}\right\rangle+2 \gamma_{m}\left\langle n^{\prime} d^{\prime}\right\rangle \\
\frac{\left\langle p^{\prime 2}\right\rangle}{d t}= & 2\left[\frac{n_{0}}{k+n_{0}}\left\langle p^{\prime 2}\right\rangle-\frac{k p_{0}}{\left(k+n_{0}\right)^{2}}\left\langle n^{\prime} p^{\prime}\right\rangle\right]-2 m_{p}\left\langle p^{\prime 2}\right\rangle \\
& -2 r\left[\frac{k_{p}^{2} p_{0} z_{0}}{\left(k_{p}^{2}+p_{0}^{2}\right)^{2}}\left\langle p^{\prime 2}\right\rangle+\frac{p_{0}^{2}}{k_{p}^{2}+p_{0}^{2}}\left\langle p^{\prime} z^{\prime}\right\rangle\right] \\
\frac{\left\langle z^{\prime 2}\right\rangle}{d t}= & 2(1-\gamma) r\left[\frac{k_{p}^{2} p_{0} z_{0}}{\left(k_{p}^{2}+p_{0}^{2}\right)^{2}}\left\langle p^{\prime} z^{\prime}\right\rangle+\frac{p_{0}^{2}}{k_{p}^{2}+p_{0}^{2}}\left\langle z^{\prime 2}\right\rangle\right]-2\left(m_{z n}+m_{z d}\right)\left\langle z^{\prime 2}\right\rangle \\
\frac{\left\langle d^{\prime 2}\right\rangle}{d t}= & 2 \gamma r\left[\frac{k_{p}^{2} p_{0} z_{0}}{\left(k_{p}^{2}+p_{0}^{2}\right)^{2}}\left\langle p^{\prime} d^{\prime}\right\rangle+\frac{p_{0}^{2}}{k_{p}^{2}+p_{0}^{2}}\left\langle z^{\prime} d^{\prime}\right\rangle\right] \\
& -2 \gamma_{m}\left\langle d^{\prime 2}\right\rangle+2 m_{z d}\left\langle z^{\prime} d^{\prime}\right\rangle+2 m_{p}\left\langle p^{\prime} d^{\prime}\right\rangle
\end{aligned}
$$


The covarinces equations of NPZD closure models are the following:

$$
\begin{aligned}
\frac{\left\langle n^{\prime} p^{\prime}\right\rangle}{d t}= & -\left[\frac{n_{0}}{k+n_{0}}\left\langle p^{\prime} z^{\prime}\right\rangle+\frac{k p_{0}}{\left(k+n_{0}\right)^{2}}\left\langle n^{\prime} z^{\prime}\right\rangle\right]+m_{z n}\left[\left\langle z^{2}\right\rangle-\left\langle n^{\prime} z^{\prime}\right\rangle\right]-m_{z d}\left\langle n^{\prime} z^{\prime}\right\rangle \\
& +\gamma_{m}\left\langle z^{\prime} d^{\prime}\right\rangle+(1-\gamma) r\left[\frac{2 k_{p}^{2} p_{0} z_{0}}{\left(k_{p}^{2}+p_{0}^{2}\right)^{2}}\left\langle n^{\prime} p^{\prime}\right\rangle+\frac{p_{0}^{2}}{k_{p}^{2}+p_{0}^{2}}\left\langle n^{\prime} z^{\prime}\right\rangle\right] \\
\frac{\left\langle n^{\prime} d^{\prime}\right\rangle}{d t}= & -\left[\frac{n_{0}}{k+n_{0}}\left\langle p^{\prime} d^{\prime}\right\rangle+\frac{k p_{0}}{\left(k+n_{0}\right)^{2}}\left\langle n^{\prime} d^{\prime}\right\rangle\right]+m_{z n}\left\langle z^{\prime} d^{\prime}\right\rangle+m_{z d}\left\langle n^{\prime} z^{\prime}\right\rangle \\
& +\gamma r\left[\frac{2 k_{p}^{2} p_{0} z_{0}}{\left(k_{p}^{2}+p_{0}^{2}\right)^{2}}\left\langle n^{\prime} p^{\prime}\right\rangle+\frac{p_{0}^{2}}{k_{p}^{2}+p_{0}^{2}}\left\langle n^{\prime} z^{\prime}\right\rangle\right]+m_{p}\left\langle n^{\prime} p^{\prime}\right\rangle+\gamma_{m}\left[\left\langle d^{\prime 2}\right\rangle-\left\langle n^{\prime} p^{\prime}\right\rangle\right] \\
\frac{\left\langle p^{\prime} z^{\prime}\right\rangle}{d t}= & -\left[\frac{n_{0}}{k+n_{0}}\left\langle p^{\prime} z^{\prime}\right\rangle+\frac{k p_{0}}{\left(k+n_{0}\right)^{2}}\left\langle n^{\prime} z^{\prime}\right\rangle\right]-\left(m_{p}+m_{z n}+m_{z d}\right)\left\langle p^{\prime} z^{\prime}\right\rangle \\
& +\frac{2 r k_{p}^{2} p_{0} z_{0}}{\left(k_{p}^{2}+p_{0}^{2}\right)^{2}}\left[(1-\gamma)\left\langle p^{2}\right\rangle-\left\langle p^{\prime} z^{\prime}\right\rangle\right]+\frac{r p_{0}^{2}}{k_{p}^{2}+p_{0}^{2}}\left[(1-\gamma)\left\langle p^{\prime} z^{\prime}\right\rangle-\left\langle z^{\prime 2}\right\rangle\right] \\
& +\left(m_{p}+m_{z n}+m_{z d}\right)\left\langle p^{\prime} z^{\prime}\right\rangle \\
\frac{\left\langle p^{\prime} d^{\prime}\right\rangle}{d t}= & -\left[\frac{n_{0}}{k+n_{0}}\left\langle p^{\prime} d^{\prime}\right\rangle+\frac{k p_{0}}{\left(k+n_{0}\right)^{2}}\left\langle n^{\prime} d^{\prime}\right\rangle\right]-m_{p}\left[\left\langle p^{\prime 2}\right\rangle-\left\langle p^{\prime} d^{\prime}\right\rangle\right]+m_{z d}\left\langle p^{\prime} z^{\prime}\right\rangle \\
& +\gamma_{m}\left\langle p^{\prime} d^{\prime}\right\rangle+\frac{2 r k_{p}^{2} p_{0} z_{0}}{\left(k_{p}^{2}+p_{0}^{2}\right)^{2}}\left[\gamma\left\langle p^{\prime 2}\right\rangle-\left\langle p^{\prime} d^{\prime}\right\rangle\right]+\frac{r p_{0}^{2}}{k_{p}^{2}+p_{0}^{2}}\left[\gamma\left\langle p^{\prime} z^{\prime}\right\rangle-\left\langle z^{\prime} d^{\prime}\right\rangle\right] \\
\frac{\left\langle z^{\prime} d^{\prime}\right\rangle}{d t}= & \frac{2 r k_{p}^{2} p_{0} z_{0}}{\left(k_{p}^{2}+p_{0}^{2}\right)^{2}}\left[(1-\gamma)\left\langle p^{\prime} d^{\prime}\right\rangle+\gamma\left\langle p^{\prime} z^{\prime}\right\rangle\right]+\frac{r p_{0}^{2}}{k_{p}^{2}+p_{0}^{2}}\left[(1-\gamma)\left\langle z^{\prime} d^{\prime}\right\rangle+\gamma\left\langle z^{\prime 2}\right\rangle\right] \\
& +m_{p}\left\langle p^{\prime} z^{\prime}\right\rangle-\left(\gamma_{m}+m_{z n}+m_{z d}\right)\left\langle z^{\prime} d^{\prime}\right\rangle
\end{aligned}
$$

These equations of mean, variances and covariance are so complex even in dimensionless form that analytic solutions are mathematically intractable. Hence, numerical simulations are carried out to observe qualitative dynamics of NPZD closure model with the following parameters choice:

$$
r=0.8, m_{p}=0.3, m_{z n}=0.01, m_{z d}=0.05, \gamma=0.25, \gamma_{m}=0.4, k=0.8, k_{p}=0.5, \beta=2.0
$$

\section{Results}

\subsection{Perturbations lead significant increase in higher trophic levels}

To observe the deviation in biomasses of higher trophic levels due to perturbation / heterogeneity, four possible scenario are considered depending on their dominance in plankton ecosystems.

1. P-dominating case where NPZD model has stable equilibrium as $p_{0}=0.4015, z_{0}=$ $0.2521, d_{0}=0.1569, n_{0}=0.1895$

2. Z-dominating case where NPZD model has stable equilibrium as $p_{0}=0.3015, z_{0}=$ $0.3521, d_{0}=0.1569, n_{0}=0.1895$

3. D-dominating case where NPZD model has stable equilibrium as $p_{0}=0.2015, z_{0}=$ $0.1521, d_{0}=0.4356, n_{0}=0.2108$ 

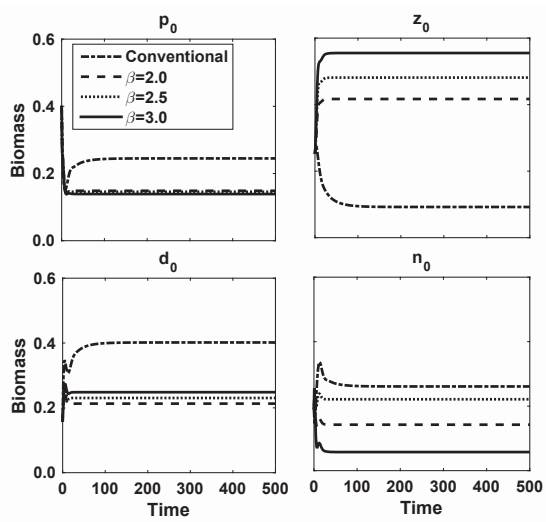
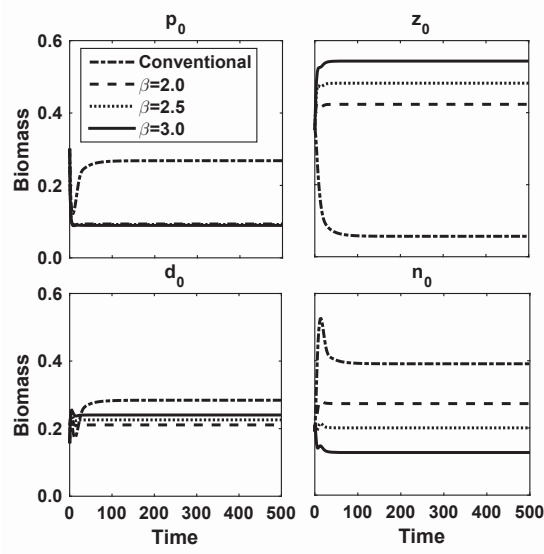

Figure 2. A significant enhancement in Z-biomass for P-dominating and Z-dominating cases. Perturbations lead higher biomass in top trophic level of NPZD system
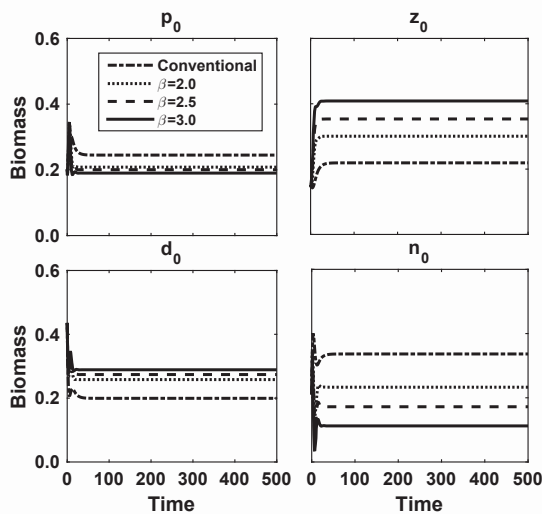
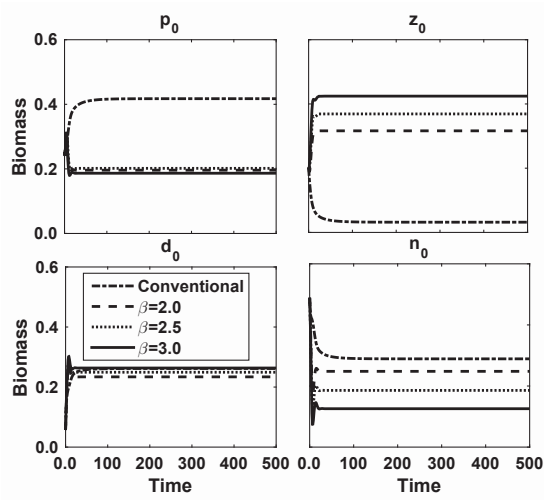

Figure 3. A significant increase in Z-biomass for $\mathrm{N}$-dominating and D-dominating cases. Perturbations support higher trophic levels of NPZD system.

4. N-dominating case where NPZD model has stable equilibrium as $p_{0}=0.2515, z_{0}=$ $0.2021, d_{0}=0.0569, n_{0}=0.4889$

These dominating cases are observed by tuning the parameter set (13) by deviating only one parameter or maximum two parameters values. In each individuals dominating cases, perturbation lead to higher biomass in zooplankton compartment (see Fig. 2 and Fig. 3). The results are robust i.e. independent of parameter choices as the changes in biomass of each plankton variables against the range of perturbations values also suggest the same (drawn in the Fig. 4 for range of parameter $r(0.2-2.0)$ and $\beta(0-5)$. A significant increment in Z-biomass is observed in closure NPZD model than conventional NPZD model. Hence, we reached at the conclusion that perturbation enhances higher trophic biomass considered in plankton models. 

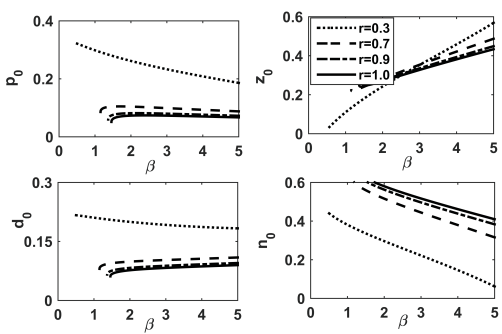
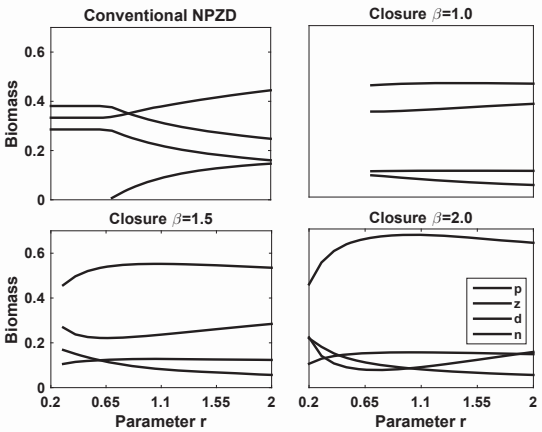

Figure 4. Due to perturbations, biomass of each components of NPZD system changes, however, a significant increase in Z-biomass is evident. Another bifurcation diagram with respect to parameter $r$ (Zgrazing rate) at different perturbations values showing variations in ecosystems components biomasses.

\subsection{Perturbations / heterogeneity (EH) and $C V_{p}$}

The statistical term coefficient of variation of phytoplanktopn $C V_{p}$ is defined as the ratio of the standard deviation against the spatial mean of phytoplankton. Using NP closure model, [6] Mandal et al. (2016) investigated that why the $C V_{p}$ varies $(0.2-4)$ with seasons and with different locations at Tokyo Bay from the observed florescence profile measured by TurboMap Laser ([4], [23]). Based on such investigations, they claimed that higher $C V_{p}$ is observed in Open Ocean than near to estuaries and lakes. Since NP model is quite simple and a basic model, the impact of heterogeneity on $C V_{p}$ is not very clear even NPZ model studied by Priyadarshi et al. 2017 only produce $C V_{p}>1$. Further, the closure NPZ model studied by Priyadarshi et al. (2017) [12] obtained a robust result $C V_{p}$ increases with heterogeneity in plankton ecosystems which is independent of parameter choices. We are interested to observe the effects of perturbations on $C V_{p}$, complexity in model increased when number of compartments in the plankton model is higher and nonlinear Holling type III grazing functional response is applied. It is observed that the highly sensitive parameters for P- biomass obtained in SA is more effective for measuring the quantity $C V_{p}$ as comparison to low sensitive parameters (see Fig. 5 \& Fig. 6 ). In the closure NPZD model at different parameter values, $C V_{p}$ increases with $\mathrm{EH}$ for all different parameter combinations shown in the Fig. 7 and Fig. 8. Perturbations lead to trophic interactions and increase the Z-biomass (higher trophic biomass). These results suggest that higher value of $C V_{p}$ indicates low production of phytoplankton (Oligotrophic region) while lower value of $C V_{p}$ indicate higher production of phytoplankton (eutrophic region). With the help of $C V_{p}$ as a measurable quantity, the highly heterogeneous and low heterogeneous locations can be identified.

\section{Discussion}

The present study has been started for the following reasons: (i) since in NP-model, heterogeneity enhances P-biomass while in NPZ model heterogeneity enhances Z-biomass and P-biomass substantially decreases with heterogeneity for some parameter sets. With these findings, no certain conclusion are derived, hence study of a bigger system NPZD model was necessary to reach at certain conclusion about impacts of heterogeneity. We were interested to know how perturbations impact on trophic interaction when complexity in plankton models increased through additional planktonic variables and parameters. These complexity 

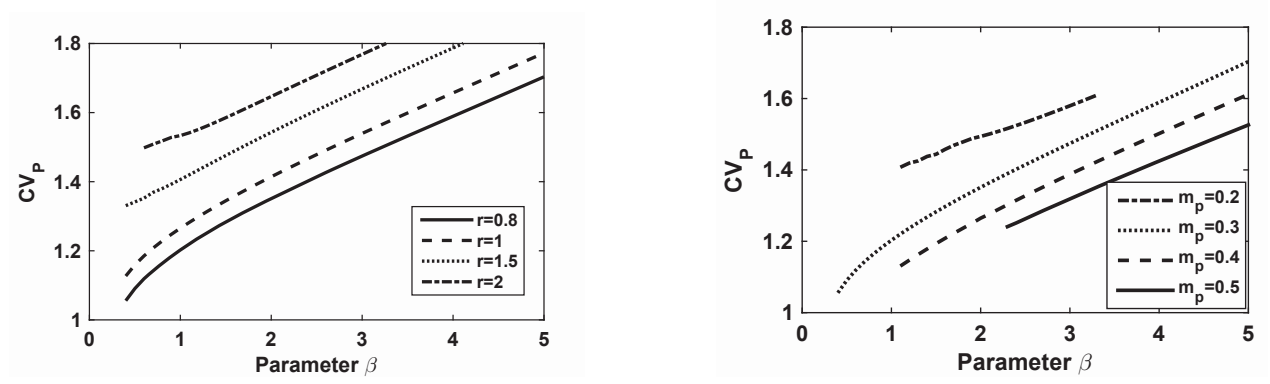

Figure 5. Perturbations in NPZD system lead higher coefficient of variation of phytoplankton $C V_{p}$ drawn at different parameters (i) $r$ and (ii) $m_{p}$. In each case, with higher perturbations higher $C V_{p}$ is quite evident.
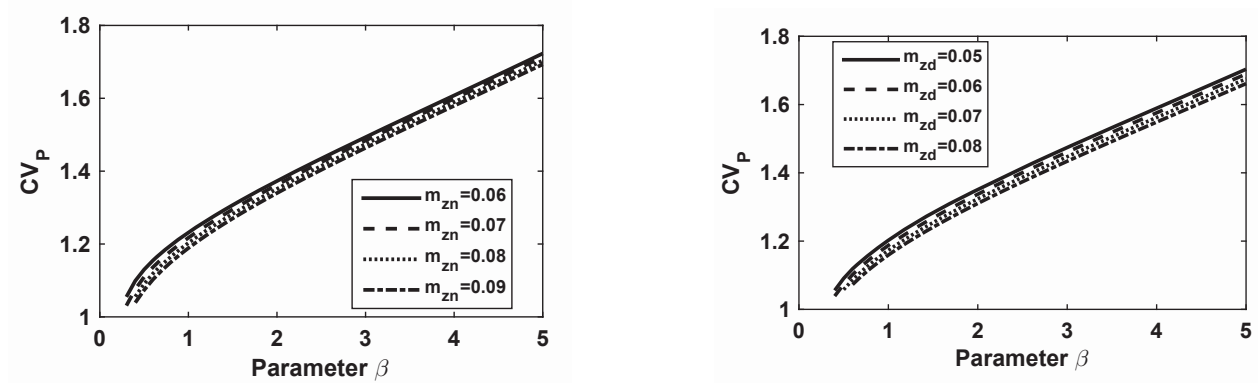

Figure 6. Perturbations in NPZD system lead higher coefficient of variation of phytoplankton $C V_{p}$ drawn at different parameters (i) $m_{z n}$ and (ii) $m_{z d}$. In each case, with higher perturbations higher $C V_{p}$ is quite evident.
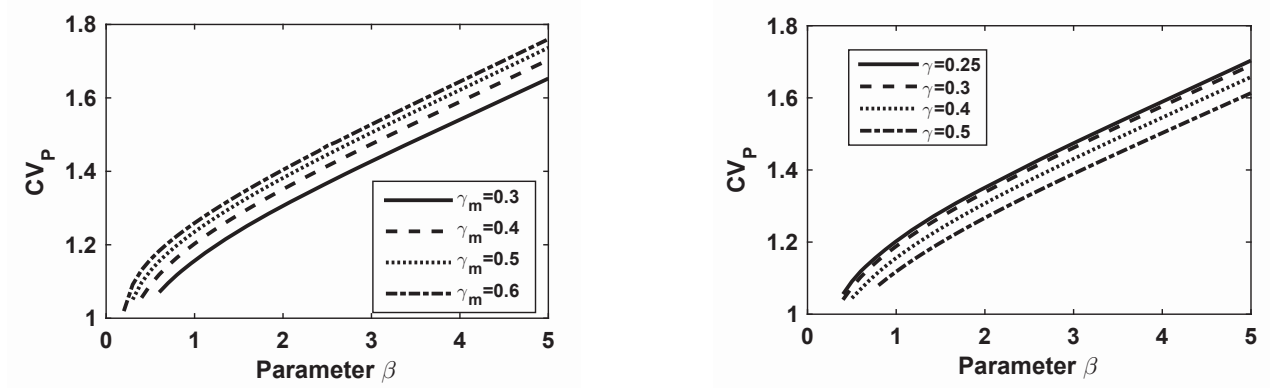

Figure 7. Perturbations in NPZD system lead higher coefficient of variation of phytoplankton $C V_{p}$ drawn at different parameters (i) $\gamma_{m}$ and (ii) $\gamma$. In each case, with higher perturbations higher $C V_{p}$ is quite evident. 

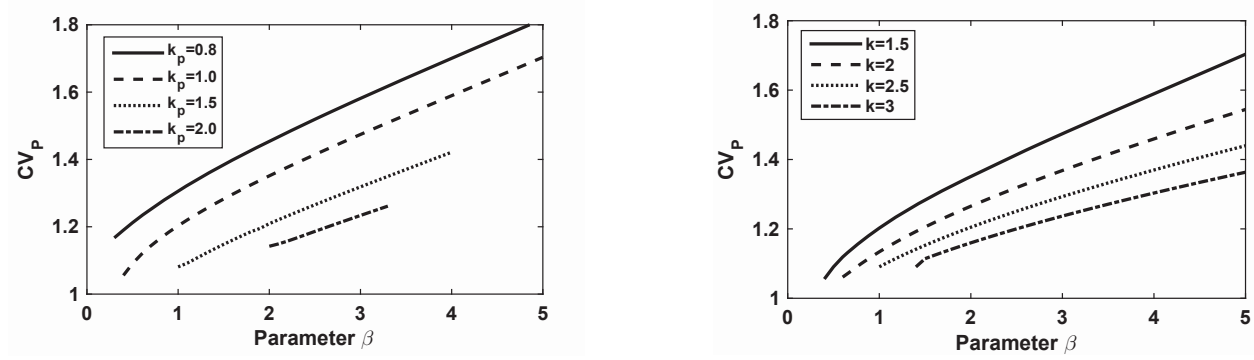

Figure 8. Perturbations in NPZD system lead higher coefficient of variation of phytoplankton $C V_{p}$ drawn at different parameters (i) $k_{p}$ and (ii) $k$. In both cases, with higher perturbations higher $C V_{p}$ is quite evident.

may affect dynamics and functioning processes of ecosystem. The NPZD model is typically assumed to be minimum model to understand plankton dynamics. From the present findings and previous findings on NP and NPZ models, we observed a robust result that heterogeneity supports higher trophic levels present in the model and may sustain bigger biodiversity. From the extensive simulations, again a robust characteristics of heterogeneity is observed i.e. Heterogeneity may increase TE and hence a positive correlation between $C V_{p}$ and TE along the trophic gradient. At higher EH, the higher TE indicates lower phytoplankton productions or higher zooplankton production while low TE indicates higher phytoplankton productions at low EH. From these results, with measurable quantity $C V_{p}$, locations can be indexed with TE (high or low). These theoretical results may be useful for understanding the dynamics of ecosystem.

\section{Conclusions}

The study suggest that perturbation may change qualitative behavior of plankton systems significantly. Heterogeneity is essential for coexistence of bigger food chain / food web as heterogeneity enhances the biomass of higher trophic levels and increase the possibility of the coexistence. The observed coefficient of variation of phytoplankton $C V_{p}$ which is the ratio of standard deviation to mean is positively associated with heterogeneity. These results are robust and hence independent of parameter choice. Thus, heterogeneity supports higher trophic levels. Based on our findings specifically for plankton systems, we conclude that The locations with high $\mathrm{CVp}$ are highly heterogeneous and have high transfer efficiency, while low $C V_{p}$ locations are less heterogeneous and have lower transfer efficiency.

\section{Acknowledgements}

The study is financially supported by the grants - UGC-BSR (R/Dev/M-14/38) and DSTSERB (R/Dev/P-07/682). The author RC also acknowledges CSIR, New Delhi India for providing Senior Research Fellowship through award letter no. 09/013(628)/2016-EMR-1 for the financial support.

\section{References}

[1] R. J. Geider, H. L. Maclntyre, and T.M. Kana, A dynamic regulatory model of phytoplanktonic acclimation to light, nutrients, and temperature, Limnology and oceanography 43(4), 679-694 (1998). 
[2] S. Diehl, S. Berger, R. Ptacnik, and A. Wild, Phytoplankton, light, and nutrients in a gradient of mixing depths: field experiments Ecology 83, 399-411 (2002).

[3] P.J. Franks and J. S. Jaffe, Microscale variability in the distributions of large fluorescent particles observed in situ with a planar laser imaging fluorometer, Journal of Marine Systems 69, 254-270 (2008).

[4] M.J. Doubell, H. Yamazaki, H. Li, and Y. Kokubu, An advanced laser-based fluorescence microstructure profiler (TurboMAP-L) for measuring bio-physical coupling in aquatic systems, Journal of plankton research 31, 1441-1452 (2009).

[5] S. Mandal, C. Locke, M. Tanaka, and H. Yamazaki, Observations and models of highly intermittent phytoplankton distributions, PloS one 9, e94797 (2014).

[6] S. Mandal, H. Homma, A. Priyadarshi, H. Burchard, S.L. Smith, K.W. Wirtz, and H. Yamazaki, A $1 D$ physical-biological model of the impact of highly intermittent phytoplankton distributions, Journal of Plankton Research 38, 964-976 (2016).

[7] S. Mandal, S.L. Smith, A. Priyadarshi, and H. Yamazaki, Micro-scale variability impacts the outcome of competition between different modelled size classes of phytoplankton, Frontiers in Marine Science 6(259) (2019).

[8] S.L. Smith, S. Mandal, A. Priyadarshi, B. Chen, and H. Yamazaki, Modeling the combined effects of physiological flexibility and micro-scale variability for plankton ecosystem dynamics, In Encyclopedia of Ocean Sciences 5, 527-535 (2019).

[9] P.J. Wallhead, A.P. Martin, and M.A. Srokosz, Spatially implicit plankton population models: transient spatial variability, Journal of Theoretical Biology 253, 405-423 (2008).

[10] A. Stein, K. Gerstner, and H. Kreft, Environmental heterogeneity as a universal driver of species richness across taxa, biomes and spatial scales, Ecology Letters 17, 866-880 (2014).

[11] C.B. Woodson and S.Y. Litvin, Ocean fronts drive marine fishery production and biogeochemical cycling, Proceedings of the National Academy of Sciences 112, 1710-1715 (2015).

[12] A. Priyadarshi, S. Mandal, S.L. Smith, and H. Yamazaki, Micro-scale variability enhances trophic transfer and potentially sustains biodiversity in plankton ecosystems, Journal of Theoretical Biology 412, 86-93 (2017).

[13] A. Priyadarshi, S. L. Smith, S. Mandal, M. Tanaka, and H. Yamazaki, Micro-scale patchiness enhances trophic transfer efficiency and potential plankton biodiversity, Scientific Reports 9(1), 1-9 (2019).

[14] Z. Yang, X. Liu, M. Zhou, D. Ai, G. Wang, Y. Wang, ... and J.T. Lundholm, The effect of environmental heterogeneity on species richness depends on community position along the environmental gradient, Scientific reports, 5(15723) (2015).

[15] A. Bar-Massada and E.M. Wood, The richness-heterogeneity relationship differs between heterogeneity measures within and among habitats, Ecography, 37(6), 528-535 (2014).

[16] W.M. Kemp, M.T. Brooks, and R.R. Hood, Nutrient enrichment, habitat variability and trophic transfer efficiency in simple models of pelagic ecosystems, Marine Ecology Progress Series 223, 73-87 (2001).

[17] A. Calbet, M.D. Agersted, S. Kaartvedt, M. Møhl, E. F. Møller, S. Enghoff-Poulsen, and D.E. Raitsos, Heterogeneous distribution of plankton within the mixed layer and its implications for bloom formation in tropical seas, Scientific Reports 5(11240) (2015).

[18] K. Dahlgren, A. Andersson, U. Larsson, S. Hajdu, and U. Båmstedt, Planktonic production and carbon transfer efficiency along a north-south gradient in the Baltic Sea, 
Marine Ecology Progress Series 409, 77-94 (2010).

[19] J.H. Steele and E.W. Henderson, The role of predation in plankton models, Journal of Plankton Research 14, 157-172 (1992).

[20] A.M. Edwards and J. Brindley, Zooplankton mortality and the dynamical behaviour of plankton population models, Bulletin of mathematical biology 61, 303-339 (1999).

[21] R. Tian, C. Chen, J. Qi, R. Ji, R.C. Beardsley, and C. Davis, Model study of nutrient and phytoplankton dynamics in the Gulf of Maine: patterns and drivers for seasonal and interannual variability, ICES Journal of Marine Science 72, 388-402 (2014).

[22] A. Yool, E.E. Popova, A.C. Coward, D. Bernie and T.R. Anderson, Climate change and ocean acidification impacts on lower trophic levels and the export of organic carbon to the deep ocean, Biogeosciences 10, 5831-5854 (2013).

[23] H. Foloni-Neto, M. Tanaka, H. Joshima, and H. Yamazaki, A comparison between quasihorizontal and vertical observations of phytoplankton microstructure, Journal of Plankton Research 38, 993-1005 (2015). 